EPJ Web of Conferences 41, 08011 (2013)

DOI: $10.1051 /$ epjconf/20134108011

(C) Owned by the authors, published by EDP Sciences, 2013

\title{
Tracing of backward energy transfer from LH1 to LH2 in photosynthetic membranes grown under high and low irradiation
}

L. Lüer, ${ }^{1}$ V. Moulisová, ${ }^{2}$ S. Henry, ${ }^{3}$ D. Polli, ${ }^{4,5}$ T. H. P. Brotosudarmo, ${ }^{3,6}$ S. Hoseinkhani, ${ }^{7}$ D. Brida, ${ }^{4}$ G. Lanzani, ${ }^{5}$ G. Cerullo, ${ }^{4}$ and R. J. Cogdell ${ }^{3}$

${ }^{1}$ IMDEA Nanoscience, 28049 Cantoblanco, Spain

${ }^{2}$ Faculty of Medicine, University of Glasgow, Glasgow G12 8QQ, United Kingdom

${ }^{3}$ Institute for Molecular Biology, University of Glasgow, Glasgow G12 8TA, United Kingdom

${ }^{4}$ CNR-IFN, Dipartimento di Fisica, Politecnico di Milano, 20133 Milan, Italy

${ }^{5}$ Italian Institute of Technology, Center forNanoScience and Technology at Politecnico di Milano, 20133 Milan, Italy

${ }^{6}$ Ma Chung Research Center for Photosynthetic Pigments, Ma Chung University, Malang 65151, Indonesia

${ }^{7}$ Department of Material Science, Università di Milano Bicocca, 20125 Milan, Italy

\begin{abstract}
By introducing derivative transient absorption spectroscopy, we obtain rate constants for backward and forward energy transfer between LH1 and LH2 complexes in purple bacterial membranes. We find that backward energy transfer is strongly reduced in membranes grown under low irradiation conditions, compared to high light grown ones. We conclude that backward energy transfer is managed actively by the bacteria to avoid LH1 exciton deactivation under high irradiation conditions. The analytical method is generally applicable to excitonically coupled systems.
\end{abstract}

\section{Introduction}

Purple bacteria are excellent model systems for investigating the basic mechanisms of photosynthetic light harvesting [1]. Typically, two light-harvesting (LH) pigment-protein complexes are present in the photosynthetic unit: LH2 contains a ring of 9 monomeric bacteriochlorophyll a (Bchl a) and 9 pairs of excitonically coupled Bchl a molecules, while LH1 contains a ring with 18 pairs of coupled $\mathrm{Bchl} \mathrm{a}$ and the reaction center ( $\mathrm{RC})$, where charge separation takes place. The increasing excitonic interaction from LH2 towards LH1 ensures efficient vectorial energy transfer (ET) towards the RC. Some species, like Rhodopseudomonas (Rps.) palustris, present different LH2 complexes if grown under high and low illumination, respectively. At low light (LL) intensity, the spectral overlap between LH1 and LH2 is much weaker than under high light (HL) conditions because in LL membranes an additional absorption band at $1.52 \mathrm{eV}$ is observed, and the absorption band at $1.45 \mathrm{eV}$ is strongly reduced.

Figure $1 \mathrm{a}$ and $\mathrm{b}$ show that the B850 low energy (L) and B875 absorption bands are so strongly superposed that backward ET from LH1 towards LH2 can be expected. It has been suggested that backward ET towards LH2 is less prominent in the LL membranes than in the HL membranes. In previous femtosecond transient absorption (TA) studies [2, 3], the contributions of forward and unrestricted use, distribution, and reproduction in any medium, provided the original work is properly cited. 
backward ET to the observed LH2 $\leftrightarrow$ LH1 equilibration dynamics could not be resolved, and therefore information on backward ET has only been obtained in an indirect and qualitative manner. In this work, we trace the real-time evolution of the exciton densities in LH2 and LH1 following selective photoexcitation. We use a specially designed femtosecond transient absorption (TA) spectrometer with $\approx 200 \mathrm{fs}$ temporal resolution, combining tunable narrowband $(\approx 10 \mathrm{meV})$ pump with broadband white-light probe pulses, and introduce an analytical method based on derivative spectroscopy for disentangling the congested transient absorption spectra of LH1 and LH2 complexes.
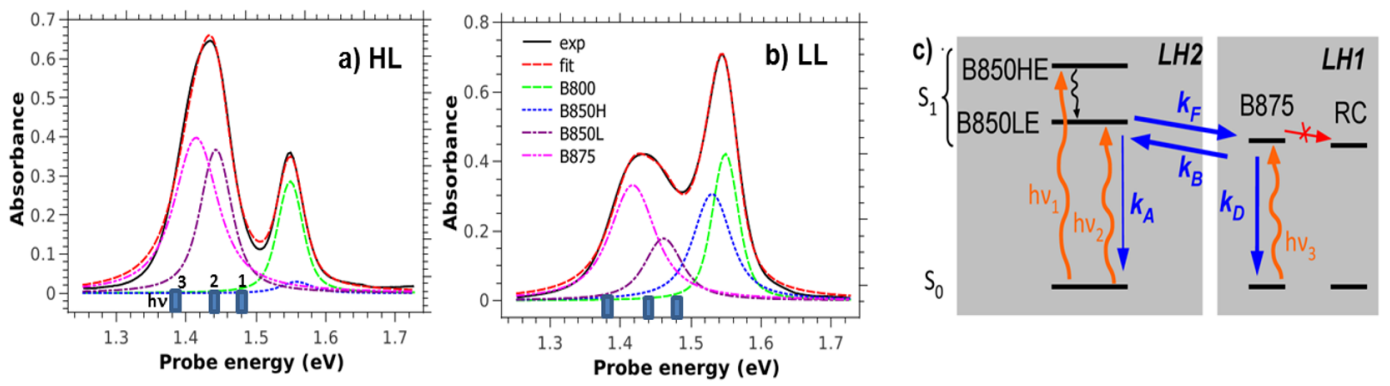

Fig. 1. Ground state absorption spectra of photosynthetic membranes grown under high (HL,a) and low (LL,b) irradiation. The positions of the narrowband pump pulses used in this work are indicated by boxes. The contributions of single absorption bands were obtained from a multi-Voigt band fitting routine. c) Photophysical model used for kinetic modelling. Please note that the bands from the isolated (B800) molecules in LH2 (with absorption at $1.54 \mathrm{eV}$ in Figs $1 \mathrm{a}, \mathrm{b}$ ) are not included in the modelling because they are not populated under our selective pumping conditions. Transfer towards RC is inhibited because membranes with closed RC were used.

\section{Results}

In Figure 2a, we show TA spectra in LL grown membranes after pumping at different photon energies. The spectra are dominated by a broad transient photobleaching (PB) around $1.37 \mathrm{eV}$, and a broad photoinduced absorption (PA) which is blue-shifted against PB. Such spectra are typical for linear or circular aggregates of excitonically coupled systems, and do not permit a clear distinction between ET and spectral relaxation within the same species. However, it is known that if the exciton delocalization is sufficiently large, then the blue-shift of PA against PB is much less that the width of both bands; in such a case, the spectral shape of the overall TA spectrum can be very well approximated by the first derivative of the ground state absorption spectrum. Consequently, the first derivative of the TA spectrum should closely resemble the second derivative of the ground state absorption spectrum. It is known that the second derivative of a bandshape function peaks at the same energy as the original function, but with strongly reduced spectral width. This property is routinely applied in $\mathrm{CW}$ optical spectroscopy ("derivative spectroscopy") in order to increase spectral selectivity.

Figure $2 \mathrm{~b}$ presents the first derivatives of the TA spectra in Figure $2 \mathrm{a}$ at various time delays. It is obvious that the derivative spectra are composed of two peaks at fixed energies, which exactly coincide with the energies of the B850L and B875 bands in Figure 1, and hence provide a measure of the populations on the LH2 and LH1 complexes, respectively. We are therefore able to unambiguously visualize ET between these two complexes. It is important to note that, while most of the population is transferred within about $10 \mathrm{ps}$, there is still significant LH2 population even after $350 \mathrm{ps}$, a clear sign of the formation of an equilibrium, which requires backward ET to occur.

In Figure $2 \mathrm{c}$ and $\mathrm{d}$, we show the population ratios $r(t)=B 850(t) /[B 850(t)+B 875(t)]$, where the single populations have been extracted from the derivative TA spectra by a multi Voigt band fitting routine. The semilogarithmic representation clearly shows that all $\mathrm{r}(\mathrm{t})$, with the exception of LL pumped at $1.385 \mathrm{eV}$, reach a constant equilibrium value between 0.35 and 0.4 . We conclude that we successfully traced the equilibration dynamics for forward and backward ET between LH2 and LH1 
complexes, and we also quantified the equilibrium itself. The availability of both equilibration dynamics and the population ratio in equilibrium allows us to unambiguously determine both forward and backward ET rate constants. We find that in LL membranes backward ET is strongly reduced with respect to HL samples [4]. In the biological context, this finding is corroborated by the fact that LH1 $\rightarrow$ LH2 backward ET allows an exciton to reach various LH1 complexes during its lifetime. This is an advantage under HL conditions (where there is a high probability that an exciton arrives at LH1 before the RC has recovered from the previous charge transfer event), but not so much under LL conditions, where the reduction of backward ET leads to a more efficient trapping of the exciton on the LH1 complex for efficient charge transfer.
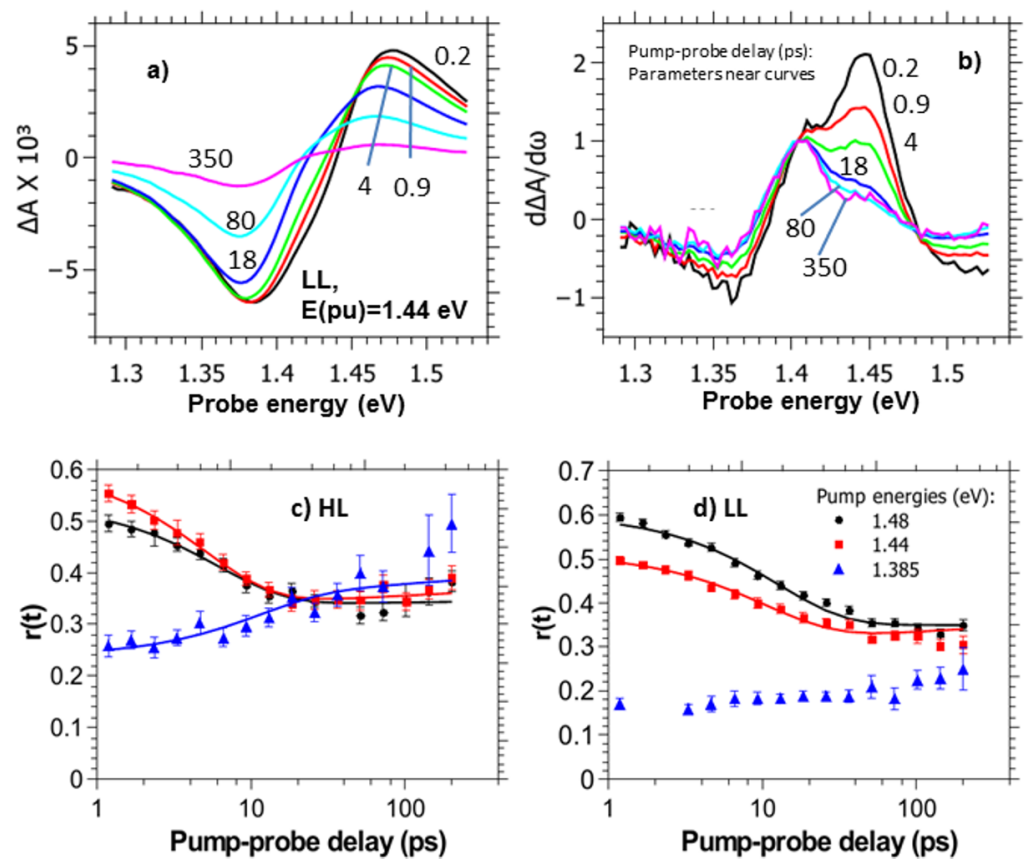

Fig. 2. a) Typical sequence of TA spectra after pumping LL membranes at $1.44 \mathrm{eV}$. b) first derivative of spectra in a). c) time-dependent ratios $r(t)=B 850(t) /[B 850(t)+B 875(t)]$ for $H L(c)$ and $L L(d)$ samples at different pumping energies. Both equilibration and the equilibrium itself can clearly be observed, a necessary condition to obtain both forward and backward ET rate constants from kinetic modelling (smooth lines), see fig. 1c.

\section{Acknowledgments}

We thank Cristian Manzoni for help with the experimental setup. R.J.C. acknowledges support from the Biotechnology and Biological Sciences Research Council; V.M., T.H.P.B., S. Hos. by the European Commission (EU Project BIMORE); L.L.: Ramon y Cajal fellow (Spanish Ministry of Science and Innovation) and EU program "AMAROUT"; D.P. and R.J.C. by the Human Frontier Science Program RGP0005; and DP by the "5 per mille junior" grant by Politecnico di Milano.

\section{References}

1. R. J. Cogdell, A. Gall, J. Köhler, Q Rev Biophys 39, 227 (2006).

2. S. Hess, et al., Proc. Natl. Acad. Sci. USA 92, 12333 (1995)

3. V. Nagarajan, W. W. Parson, Biochemistry 36, 2300 (1997).

4. L. Lüer, V. Moulisová, S. Henry, D. Polli, T. Brotosudarmo, S. Hoseinkhani, D. Brida, G. Lanzani, G. Cerullo, R. Cogdell, Proc. Natl. Acad. Sci. USA 109, 1473 (2012). 